DRAFT VERSION JUNE 20, 2021

Preprint typeset using LATEX style AASTeX6 v. 1.0

\title{
AN EXPLOSION IS TRIGGERED BY THE LATE COLLAPSE OF THE COMPACT REMNANT FROM A NEUTRON STAR MERGER
}

\author{
ANTONIOS NATHANAIL \\ Institut für Theoretische Physik, Goethe Universität Frankfurt, \\ Max-von-Laue-Str.1, 60438 Frankfurt am Main, Germany; nathanail@th.physik.uni-frankfurt.de
}

\begin{abstract}
It is known that a binary neutron star merger produces a hypermassive neutron star. The lifetime of this compact remnant depends on the total mass and the equation of state. The collapse of this compact remnant to a blackhole-torus system is expected to give rise to a powerful jet and a short gamma-ray burst. Nevertheless, if the collapse is delayed half a second or so, the surrounding matter would be already accreted and/or expelled and hence no significant torus is formed. However, the collapse itself gives rise to a quasi-isotropic magnetized fireball. This magnetic bomb dissipates much of its energy due to magnetic re-connection and produces the prompt emission. The energy range of such an explosion depends on the initial magnetic field strength and the amplification of the magnetic energy during merger. We briefly estimate the physical parameters at the time of collapse. We discuss the production of a quasi-isotropic magnetized fireball and its subsequent interaction with the ejected matter during merger, as the outcome of the coalescence of a binary neutron star system. We further suggest the radial stratification of the outflow, following the quasi-normal modes of the black hole.
\end{abstract}

Keywords: gravitational waves; gamma-ray bursts

\section{INTRODUCTION}

The dawn of the multi-messenger era was marked by the observation of GW170817 and GRB170817A, with afterglow detection across the electromagnetic (EM) spectrum (The LIGO Scientific Collaboration \& The Virgo Collaboration 2017; The LIGO Scientific Collaboration et al. 2017). The simultaneous detection of gravitational waves $(\mathrm{GW})$ and a short gamma-ray burst (GRB) made it clear that at least some shortGRBs are produced by binary neutron star mergers (BNS). It was a long standing conjecture that BNS are progenitors of short-duration GRBs (Eichler et al. 1989; Narayan et al. 1992).

One point of extreme interest is the overall low energetics of the prompt emission and the faint gamma-ray pulse, compared to the canonical short-GRB which pictures a highly relativistic outflow. After the merger of the BNS a hyppermassive neutron star (HMNS) is produced. The usual picture needs the collapse of this HMNS to produce a black hole and together with the surrounding torus to give rise to a relativistic jet expected. However, the isotropic energy of GRB170817A observed to be $\sim 10^{46} \mathrm{erg}$. The main arguments for this low flux detection is picturing radiation from an off-axis jet, or radiation coming from a cocoon produced by the jet while drilling through the ejected matter

1 Numerical simulations of BNS systems, with a total mass above $3.2 M_{\odot}$, have shown that after the merger, a magnetic-jet structure from the black-hole-torus system is produced (Rezzolla et al. 2011; Ruiz et al. 2016; Kawamura et al. 2016). This is the starting point for a jet from a BNS merger (Aloy et al. 2005) and for off-axis radiation (Lazzati et al. 2017a,b; Kathirga-
The first to consider the interaction of a jet and the BNS ejecta was Nagakura et al. (2014) and Murguia-Berthier et al. (2014). They incorporated a density profile, inspired from BNS simulations, mimicking the BNS ejecta and the subsequent drilling of the jet through the ejecta. These can provide jet conditions that allow the outflow to break out from the ejecta or not.

In what follows, we describe the outcome of a BNS merger, where the merger remnant has a lifetime of some seconds (even a fraction of a second). At the time of the collapse the surrounding matter consists a negligible torus. Following this path, no jet is expected to form. We discuss the collapse of the merger remnant as the central engine of a magnetic explosion that drives a short-GRB. Most of the amplified, during merger, magnetic energy is released within a millisecond during collapse. This forms a magnetised fireball.

In section 2 we estimate the physical parameters of the merger remnant and the surrounding torus at $\sim 1$ s, then present the main features of the collapse and discuss the energetics of such explosions. In section 3 we discuss the imprint of this explosion. In section 4 we conclude.

\section{THE OUTCOME OF THE MERGER AND THE COLLAPSE OF THE SMNS}

The outcome of the coalescence of a BNS can have different paths, which in turn would have different observational imprints. The difference in the collapse time of the HMNS,

maraju et al. 2018) and coccon emission (Gottlieb et al. 2018). 


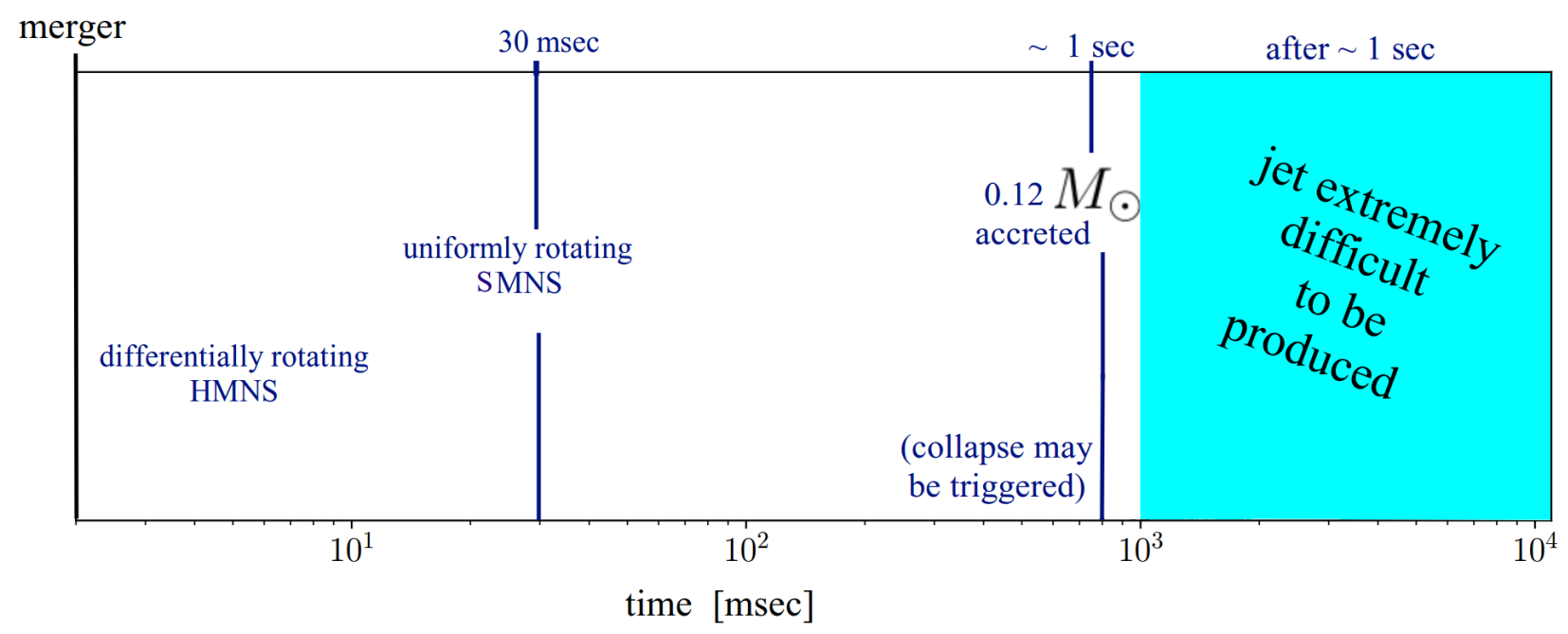

Figure 1: We show the lifetime of the SMNS, if collapse is triggered after $\sim 1$ s, then it is extremely difficult to produce a jet.

or a stable supra-massive neutron star (SMNS) ${ }^{2}$ configuration could explain the short-GRB phenomenology (Rezzolla \& Kumar 2015; Ciolfi \& Siegel 2015; Usov 1992; Metzger et al. 2008).

Electromagnetic outputs of merging objects have been considered in the literature. Especially after the collapse of the SMNS, where the strong magnetic field interaction with the black hole that lasts a considerable amount of time, can shape the afterglow of a short-GRB (Lyutikov 2011). In this paper we focus on the possibility that the SMNS produced during merger, collapses in a few seconds after merger. The important point is not exactly the lifetime of the SMNS itself, but the parameters of the torus surrounding it. The matter of the collapsing star itself quickly hides behind an event horizon, but the magnetic energy stored in the near zone magnetosphere of the SMNS is released in a millisecond scale producing an outward magnetic shock. The magnetic energy of the SMNS is expected to be more than $10^{50} \mathrm{erg}$, due to magnetic field amplification during merger. Another meaningful point to note, is the difference from the collapse of an isolated NS which is expected to give rise to a short radio transient (Falcke \& Rezzolla 2014). The major differences are two, on one hand the enhanced magnetic energy due to amplification during merger, and on the other hand the fact, that even if no torus is formed, the SMNS is not isolated. It rather lives inside a dust of left-over matter. It has been argued that this pollution may not allow the magnetar model to function (Murguia-Berthier et al. 2014). It is exactly this baryon pollution that contribute to the production of a fireball. Lehner et al. (2012) proposed that the collapse of the SMNS can produce an electromagnetic transient comparable in energy with a jet from a black-hole-torus when the SMNS collapses, and could contribute to the production of a short-GRB. They per-

\footnotetext{
${ }^{2}$ A SMNS is a NS above the TOV maximal mass which can still be supported by rigid rotation.
}

formed simulations for the gravitational collapse of an isolated magnetized neutron star and showed the instantaneous (millisecond scale) dissipation of the magnetic energy. They further discussed in detail how this picture would evolve in the presence of an accretion disc, which is the case for the remnant of a BNS merger. They compared the electromagnetic luminosity prior to merger, with the post merger phase were the SMNS has a large but not coherent magentic field. Then, they estimated the electromagnetic transient produced from the collapse of the merger remnant and compared with the subsequent jet produced by the trapped magnetic flux between the newly formed black hole and the accretion disc (Lehner et al. 2012).

Following this thinking and these estimations, we show that if the lifetime of the SMNS is longer than a second or so, then the limited amount of mass of the torus cannot result in a jet, but the collapse of the SMNS alone will power the short-GRB.

The total mass (where in the case of GW170817 was $2.74 M_{\odot}$ The LIGO Scientific Collaboration \& The Virgo Collaboration (2017)) and the EOS play the major role for the path that the merger product will take. Magnetohydrodynamic turbulence resulting in a effective viscosity can account for angular momentum transport which would leave a uniformly rotating SMNS after $\sim 30 \mathrm{msec}$. Estimating this viscous angular-momentum transport yields

$$
\begin{array}{r}
t_{\mathrm{MRI}} \sim \frac{R^{2}}{\nu} \sim 30 \mathrm{msec} \\
\left(\frac{R}{15 \mathrm{~km}}\right)^{2}\left(\frac{H}{7.5 \mathrm{~km}}\right) \\
\times\left(\frac{\alpha}{0.01}\right)^{-1}\left(\frac{c_{s}}{c / 3}\right)^{-1},
\end{array}
$$

where $\nu=\alpha c_{s} H$ is the viscous parameter, $c_{s}$ the speed of sound, $H$ the typical vertical scale height and the $\alpha$ parameter (Shakura \& Sunyaev 1973). Thus, the SMNS is uniformly rotating in less than $30 \mathrm{msec}$ (Fujibayashi et al. 2017) ${ }^{3}$, whereas

\footnotetext{
${ }^{3}$ The ADM mass of the SMNS in this study is $\sim 2.65 M_{\odot}$, and thus is
} 
the outer part constitute a quasi-keplerian disk (Hanauske et al. 2017).

The next important step is to estimate the evolution of the SMNS and the surrounding torus. If the HMNS survives for $30 \mathrm{msec}$, then differential rotation is lost and the maximum mass can be estimated for a uniformly rotating star. However, it is important to take into account the extra thermal pressure which can be $2 \%$ of the total, especially in the first stages after merger. The cooling of the SMNS due to neutrino diffusion can be estimated:

$$
t_{\nu}^{\text {diff }} \simeq 3 \tau_{\nu} \frac{\Delta R}{c} \sim 0.8 \mathrm{~s}\left(\frac{\tau_{\nu}}{10^{3}}\right)\left(\frac{\Delta R}{20 \mathrm{~km}}\right),
$$

where $\Delta R$ is the local density scale height and $\tau_{\nu}$ is the optical depth, with a mean value of $10^{3}$ for neutrinos of $10-100 \mathrm{MeV}$ (Dessart et al. 2009). This means that in around 1 s there is a $2 \%$ reduction of pressure, which could trigger the collapse of the SMNS. However, a study from Kaplan et al. (2014) showed that models with more thermal support are less compact, which make them more stable in a longer timescale.

At this time the structure of the surrounding torus has also changed significantly. The viscous accretion timescale estimated for the torus:

$$
\begin{aligned}
t_{a c c r} \simeq 1 \mathrm{~s} & \left(\frac{R_{T}}{50 \mathrm{~km}}\right)^{2}\left(\frac{H_{T}}{25 \mathrm{~km}}\right)^{-1} \\
& \times\left(\frac{\alpha}{0.01}\right)^{-1}\left(\frac{c_{s}}{0.1 c}\right)^{-1}
\end{aligned}
$$

where $R_{T}$ is the radius of the torus and $H_{T}$ is the typical vertical scale height of the torus. Then, the mass accretion rate onto the SMNS yields

$$
\begin{aligned}
\dot{M}_{S M N S} \simeq \frac{M_{T}}{t_{a c c r}} \sim 0.2 & M_{\odot} s^{-1}\left(\frac{\alpha}{0.01}\right)\left(\frac{M_{T}}{0.2 M_{\odot}}\right) \\
& \times\left(\frac{R_{T}}{50 \mathrm{~km}}\right)^{-2}\left(\frac{H_{T}}{25 \mathrm{~km}}\right),
\end{aligned}
$$

where $M_{T}$ is the mass of the torus. However, this accretion rate is not stationary, since the mass of the torus decreases in time and the torus expands. The radius of the torus can reach $140 \mathrm{~km}$ in $1 \mathrm{~s}$. Nevertheless, it can account for a mass accretion of $\sim 0.12 M_{\odot}$ in 1s (Fujibayashi et al. 2017).

The previous estimate is another indication that collapse could be triggered at around $1 \mathrm{~s}$ after merger, due to accretion ${ }^{4}$. Furthermore, the effective viscosity inside the torus results in the expansion of the torus. The density in the vicinity of the SMNS, at the time of collapse, is extremely important. As we show below, this is the parameter that designates the outcome of the collapse, a black-hole-torus magnetic jet or an induced magnetic explosion. The density of the torus at $1 \mathrm{~s}$ is estimated

\footnotetext{
relevant to the BNS that produced GW170817.

4 As an example, for the LS220 EOS an equal mass BNS of total mass $M=2.87 M_{\odot}$, collapses straight after merger, whereas for a total mass $M=2.67 M_{\odot}$ does not collapse for some tens of milliseconds (Bovard et al. 2017).
}

by its left over mass and its expanded radius:

$$
\begin{aligned}
\rho_{T} \simeq \frac{M_{T}}{2 H_{T} \pi R_{T}^{2}} & \sim 9.2 \times 10^{9} \mathrm{~g} / \mathrm{cm}^{3}\left(\frac{M_{T}}{0.08 M_{\odot}}\right) \\
& \times\left(\frac{R_{T}}{140 \mathrm{~km}}\right)^{-2}\left(\frac{H_{T}}{70 \mathrm{~km}}\right)^{-1},
\end{aligned}
$$

where all quantities are for the expanded torus at $1 \mathrm{~s}$ after merger. We should point out here, that if the collapse of the SMNS occurs even later, then there is the possibility that no debris disk is formed at all (Margalit et al. 2015).

Before discussing about the production of a jet or a magnetic explosion, we should first have an estimate of the mean magnetic field strength of the SMNS. It is expected that during the merger process small scale turbulence can amplify the magnetic field in values higher than $10^{16} G$ (Rasio \& Shapiro 1999; Zrake \& MacFadyen 2013; Giacomazzo et al. 2015). In such extreme conditions the magnetic energy can reach as high as $\sim 10^{51} \mathrm{erg}$ Kiuchi et al. (2015).

We check what happens, if the SMNS collapses after 1s. One of the most important points, is the condition for the establishment of a magnetic jet. A stable configuration to be built by the black-hole-torus system needs that, at least, the torus pressure can balance the magnetic pressure. Following the above discussion, we assume that the mean magnetic field of the SMNS is $B \simeq 3 \times 10^{16} G$. This yields:

$$
\begin{array}{r}
\frac{B_{S M N S}^{2}}{8 \pi} \simeq 3.5 \times 10^{31} \mathrm{dyn} / \mathrm{cm}^{2}\left(\frac{B_{S M N S}}{3 \times 10^{16} G}\right)^{2} \\
\gg 9.2 \times 10^{29} \mathrm{dyn} / \mathrm{cm}^{2}\left(\frac{\rho_{T}}{9.2 \times 10^{9} \mathrm{~g} / \mathrm{cm}^{3}}\right) \simeq \rho_{T} c^{2} .
\end{array}
$$

This is estimated at around $1 \mathrm{~s}$ after merger. It is evident that at later times, when the torus has expanded more, the density decreases and the establishment of a magnetic jet becomes more difficult. A lower estimate we get from the accretion rate at $1 \mathrm{~s}$, which is $\sim 0.02 M_{\odot} s^{-1}$ as reported in Fujibayashi et al. (2017). This yields:

$$
B_{S M N S}^{2} / 8 \pi \gg 2.6 \times 10^{28} \mathrm{dyn} / \mathrm{cm}^{2} \sim \dot{M} c / 4 \pi r_{B H}^{2} .
$$

The above discussion is summarized in figure 1 . So far, we have estimated that if the collapse is triggered around or after $\sim 1$ s after merger, the magnetic energy of the SMNS is released and induce a powerfull explosion of $E_{\text {exp }} \sim 10^{51} \mathrm{erg}$, contrary to a magnetic jet expected. The energetics for this magnetic bomb follows from the properties of the remnant (SMNS) itself. The absence of a jet was discussed by Salafia et al. (2018), where instead a flare produced during the magnetic field amplification, gave rise to a relativistic isotropic fireball, which could explain the prompt emission of GRB170817A (Salafia et al. (2017), see also ?). Whereas, what we discussed here comes after merger and the absence of a jet is due to the physical conditions that come around or after $\sim 1 \mathrm{~s}$. 


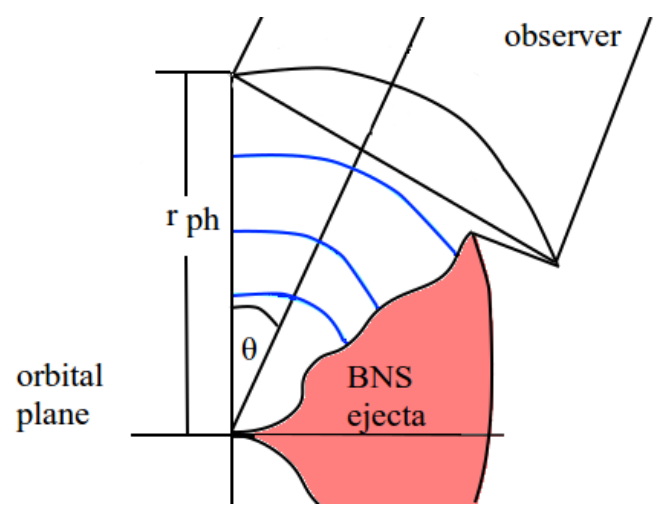

Figure 2: In higher latitudes, the low density region allow the quasi-spherical fireball to escape and reach the transparency radius, $r_{p h}$. With blue color, we depict the slower cells of the fireball produced by the "ring-down" of the black hole.

The amount of baryons in this magnetic bomb is subject to the leftover matter exterior the SMNS and the neutrinodriven wind. Very quickly this magnetic bomb catches up the relatively slow moving ejecta and crash on to them. This matter, expelled dynamically or secularly, is responsible for the appearance of a kilonova (Abbott et al. 2017) and moves with velocities $u \simeq 0.1-0.2 c$ (Metzger et al. 2010; Bovard et al. 2017).

The matter ejected during merger depends on the EOS, nevertheless its averaged distribution on a sphere far from the merger point shows that above the equator the overall ejected matter may be three orders of magnitude less than what was ejected in the equatorial plane (roughly $\sim 10^{-6} M_{\odot}$, Bovard et al. (2017)). The magnetic bomb finds it easier to pass through the ejecta from higher latitudes. This means that it may have a wide angle jet structure, or rather that it is quasiisotropic with a big opening angle from the orbital axis.

We have to stress out that what was discussed in this section is not at all any kind of a proof that the collapse of the merger remnant came at $\sim 1 \mathrm{~s}$ after merger. The major point here was that if the collapse comes after $\sim 1 \mathrm{~s}$, then it is extremely difficult to produce a magnetic jet.

\section{THE IMPRINT OF THE MAGNETIC BOMB}

The formation of a magnetised fireball that crashes on the ejecta is established, if the collapse of the SMNS happens at $\sim 1 \mathrm{~s}$. In this section we discuss the evolution of this magnetic bomb. We show that after the explosion a relativistic outflow is produced. We further discuss how this outflow can push matter from the polar region and surpass the BNS ejecta. The amplified magnetic field in the SMNS is favored in the toroidal direction and could produce a magnetically driven plasma gun (Contopoulos 1995). At the time of collapse all this energy is released in a millisecond scale. This explosion induces a shock to the surrounding matter. The shock front is perpendicular to the radial direction and par-

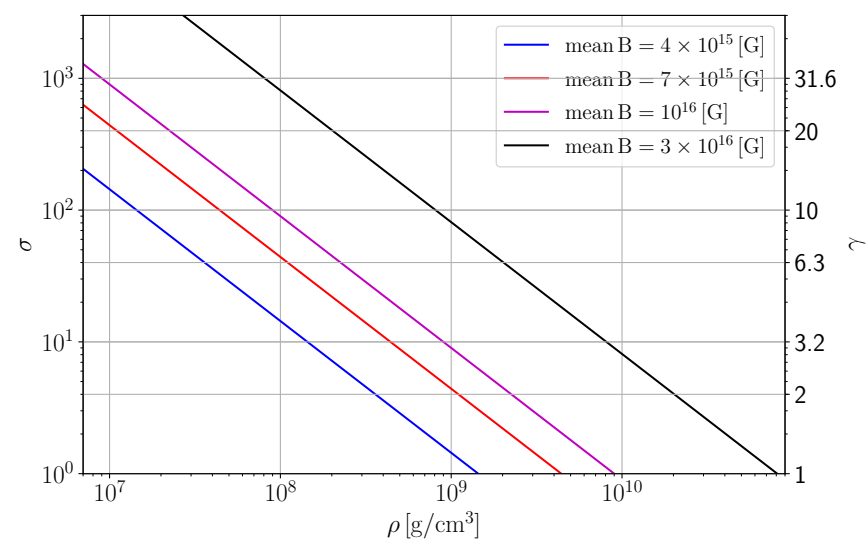

Figure 3: The magnetization parameter $\sigma$ (left column) and the Lorentz factor $\gamma$ (right column), that corresponds to such magnetization in the downstream region, for the relevant values of the density $\rho$, for different values of the mean magnetic field $B$.

allel to the predominantly toroidal magnetic field. We assume that the upstream pressure is small, since the SMNS has collapsed when the magnetic explosion is produced. Analysis of the jump conditions across the shock can yield useful estimations for the parameters of the post-shock region and have been widely used for astrophysical purposes (e.g. Kennel \& Coroniti (1984)). We define the magnetization parameter as:

$$
\sigma=\frac{B^{2}}{4 \pi \rho c^{2}}
$$

then the corresponding downstream Lorentz factor is

$$
\gamma \simeq \sqrt{\sigma}
$$

In figure 3 we plot the magnetization parameter for the relevant values of the magnetic field and the density, where the density values can be viewed as an angular distribution of the matter from the equator till the polar region, where density falls significantly. It is evident, that for a polar density of $10^{7} \mathrm{~g} / \mathrm{cm}^{3}$ (as reported in Fujibayashi et al. (2017)) an outflow with a Lorentz factor of 10 is formed.

The next question to address, is whether this outflow can break out form the ejecta. In the case of a relativistic flow interacting with an external medium, the Sedov length signals the radius where deceleration begins. The Sedov length is generally defined as the radius where the energy of the swept up matter: $E=m_{p} c^{2} \int_{0}^{l} 4 \pi \rho r^{2} d r$, equals the energy of the explosion. The polar regions with less density are favored as a pathway for the explosion. As mentioned before, the ejected mass from the polar regions is on the the order of $\sim$ $10^{-6} M_{\odot}$, (Bovard et al. 2017). Thus, the rest mass energy of the matter in the polar region is $E_{\text {mpolar }} \sim 10^{48} \mathrm{erg}$. Since the energy of the explosion is $E_{\text {exp }} \sim 10^{51} \mathrm{erg}$, the deceleration radius is reached further out in the inter-stellar medium. and the outflow can successfully surpass the BNS ejecta from the polar region. 
The prompt gamma-ray emission consists of a non-thermal pulse produced by accelerated particles through magnetic reconnection that will follow the collapse and the release of the magentic energy of the SMNS. During the expansion of the fireball, further dissipation of the magnetic energy can contribute more to this. This will be followed (or accompanied) by a thermal high energy pulse produced almost with the fireball itself due to the pressure and the high temperatures in the post shock region. When the fireball reaches the photosphere $\left(r_{p h} \sim 10^{12} \mathrm{~cm}\right)$, it is already further beyond the slow moving ejecta (at a similar time the ejecta is almost an order of magnitude closer to the source) and thus photons will not find any other obstacle(fig. 2).

The duration of the high-energy pulse, which in the case of GRB170817A was $\sim 2$ s (Goldstein et al. 2017), The duration of the emission from the spherical cap is $d t \sim h / c=r_{p h} \times$ $(1-\cos (\theta)) / c \simeq 2 r_{p h} \theta_{f}^{2} / c$. For a mildly relativistic outflow with $\Gamma>1 / \theta_{f}$, the relevant timescale of the pulse is $d t \sim$ $r_{p h} / 2 c \Gamma^{2}$ (Piran 2005), which for a range of $\Gamma \simeq 7-10$, the duration is $d t \sim 1-2 \mathrm{~s}$. We should also mention here that even in the canonical short-GRB picture the $\sim 2$ s duration may be a problem if the disk is not massive enough and is accreted in less than a second.

Another point that we want to touch is a way of radially stratifying the fireball. It is known that the production of a black hole is followed by the "ringing down" of the black hole (Kokkotas \& Schmidt 1999). The first burst from the collapse is followed by further pulses with almost an order of magnitude less intensity and a millisecond duration (Lehner et al. 2012; Dionysopoulou et al. 2013; Most et al. 2018). Every pulse will be accompanied by a production of a slower fireball, due to the reduced magnetic pressure injected by the pulse. The quasi-normal modes of the black hole are exponentially decaying, but can contribute to the radial stratification of the fireball. The fireball may also form a coccoon-like structure at the intersection with the high-density ejecta and may have a similar EM signature with a coccoon produced by a jet (Gottlieb et al. 2018).

There is no need to speculate more about the radiation imprint of such fireball, since this should follow from a complete study of such a process. Nevertheless, we should add that several observational facts that followed GW170817 and GRB170817A can accommodate the production of such wide-angle mildly relativistic outflow. The rising X-ray emission ( $\sim 3$ days after the event) and the radio observations ( $\sim 20$ days) can be explained with a quasi-isotropic outflow, and in the case we discussed the kinetic energy can be up to the order of $10^{50} \mathrm{erg}$ (Alexander et al. 2017; Margutti et al. 2017). Furthermore, it was stated that the observed radio data have no direct indication of the presence of a jet, and they can be explained by a wide-angle mildly relativistic outflow (Mooley et al. 2018). Observations (radio, optical and X-ray) cannot rule out one or the other choice (Margutti et al. 2018).

\section{CONCLUSIONS}

The aim of this note is to present and discuss the possibility that the outcome of a BNS merger follows a path that produces a quasi-spherical fireball behind the BNS ejecta rather than a jet. The main outcome of a merger discussed in the literature is the production of a black hole together with a surrounding torus or a stable magnetar, both of them are used in order to explain short-GRBs. In light of the new observations, we propose that it is equally possible to follow a third path, namely that the compact remnant, produced after merger, collapses and no significant torus is produced. This has as a consequence that all the magnetic energy of the SMNS will be released in a millisecond scale. The important point here, is that this energy can be as much as $10^{50} \mathrm{erg}$, due to magnetic field amplification during merger. Thus, energetically it could in principle power a short-GRB (Fong et al. 2015).

\section{ACKNOWLEDGEMENTS}

It is a pleasure to thank D. Giannios, C. Fromm, O. Porth and L. Rezzolla for useful discussions. The author is supported by an Alexander von Humboldt Fellowship.

\section{REFERENCES}

Abbott B. P., et al., 2017, Astrophys. J. Lett., 850, L39

Alexander K. D., et al., 2017, Astrophys. J. Letters, 848, L21

Aloy M. A., Janka H., Müller E., 2005, Astron. Astrophys., 436, 273

Bovard L., Martin D., Guercilena F., Arcones A., Rezzolla L., Korobkin O., 2017, Phys. Rev. D, 96, 124005

Ciolfi R., Siegel D. M., 2015, Astrophys. J., 798, L36

Contopoulos J., 1995, Astrophys. J., 450, 616

Dessart L., Ott C. D., Burrows A., Rosswog S., Livne E., 2009, Astrophys, J., 690, 1681

Dionysopoulou K., Alic D., Palenzuela C., Rezzolla L., Giacomazzo B., 2013, Phys. Rev. D, 88, 044020

Eichler D., Livio M., Piran T., Schramm D. N., 1989, Nature, 340, 126

Falcke H., Rezzolla L., 2014, Astron. Astrophys., 562, A137

Fong W., Berger E., Margutti R., Zauderer B. A., 2015, Astrophys. J., 815, 102

Fujibayashi S., Sekiguchi Y., Kiuchi K., Shibata M., 2017, Astrophys. J., 846, 114

Giacomazzo B., Zrake J., Duffell P. C., MacFadyen A. I., Perna R., 2015, Astrophys. J., 809, 39

Goldstein A., et al., 2017, Astrophys. J. Letters, 848, L14

Gottlieb O., Nakar E., Piran T., 2018, Mon. Not. R. Astron. Soc., 473, 576

Hanauske M., Takami K., Bovard L., Rezzolla L., Font J. A., Galeazzi F.,

Stöcker H., 2017, Phys. Rev. D, 96, 043004

Kaplan J. D., Ott C. D., O’Connor E. P., Kiuchi K., Roberts L., Duez M., 2014, Astrophys. J., 790, 19

Kathirgamaraju A., Barniol Duran R., Giannios D., 2018, Mon. Not. R Astron. Soc., 473, L121

Kawamura T., Giacomazzo B., Kastaun W., Ciolfi R., Endrizzi A., Baiotti L., Perna R., 2016, Phys. Rev. D, 94, 064012

Kennel C. F., Coroniti F. V., 1984, Astrophys. J., 283, 694

Kiuchi K., Sekiguchi Y., Kyutoku K., Shibata M., Taniguchi K., Wada T., 2015, Phys. Rev. D, 92, 064034

Kokkotas K. D., Schmidt B. G., 1999, Living Rev. Relativ., 2, 2

Lazzati D., Deich A., Morsony B. J., Workman J. C., 2017a, Mon. Not. R. Astron. Soc., 471, 1652 
Lazzati D., López-Cámara D., Cantiello M., Morsony B. J., Perna R., Workman J. C., 2017b, Astrophys. J. Letters, 848, L6

Lehner L., Palenzuela C., Liebling S. L., Thompson C., Hanna C., 2012, Phys. Rev. D, 86, 104035

Lyutikov M., 2011, Phys. Rev. D, 83, 124035

Margalit B., Metzger B. D., Beloborodov A. M., 2015, Phys. Rev. Lett., 115, 171101

Margutti R., et al., 2017, Astrophys. J. Letters, 848, L20

Margutti R., et al., 2018, Astrophys. J. Letters, 856, L18

Metzger B. D., Quataert E., Thompson T. A., 2008, Mon. Not. R. Astron. Soc., 385, 1455

Metzger B. D., et al., 2010, Mon. Not. R. Astron. Soc., 406, 2650

Mooley K. P., et al., 2018, Nature, 554, 207

Most E. R., Nathanail A., Rezzolla L., 2018, preprint, (arXiv: 1801.05705)

Murguia-Berthier A., Montes G., Ramirez-Ruiz E., De Colle F., Lee W. H., 2014, Astrophys. J., 788, L8

Nagakura H., Hotokezaka K., Sekiguchi Y., Shibata M., Ioka K., 2014, Astrophys. J., 784, L28
Narayan R., Paczynski B., Piran T., 1992, Astrophys. J. Lett., 395, L83

Piran T., 2005, Reviews of Modern Physics, 76, 1143

Rasio F., Shapiro S., 1999, Class. Quantum Grav., 16, R1

Rezzolla L., Kumar P., 2015, Astrophys. J., 802, 95

Rezzolla L., Giacomazzo B., Baiotti L., Granot J., Kouveliotou C., Aloy M. A., 2011, Astrophys. J. Letters, 732, L6

Ruiz M., Lang R. N., Paschalidis V., Shapiro S. L., 2016, Astrophys. J. Lett., 824, L6

Salafia O. S., Ghisellini G., Ghirlanda G., Colpi M., 2017, preprint, (arXiv:1711.03112)

Salafia O. S., Ghisellini G., Ghirlanda G., 2018, Mon. Not. R. Astron. Soc., 474, L7

Shakura N. I., Sunyaev R. A., 1973, Astron. Astrophys., 24, 337

The LIGO Scientific Collaboration The Virgo Collaboration 2017, Phys. Rev. Lett., 119, 161101

The LIGO Scientific Collaboration et al., 2017, Astrophys. J. Lett., 848, L12 Usov V. V., 1992, Nature, 357, 472

Zrake J., MacFadyen A. I., 2013, Astrophys. J., 769, L29 